\title{
Hole Free Phase Plate Electron Tomography in Material Sciences
}

\author{
Misa Hayashida $^{1}$, Amin Morteza Najarian ${ }^{1,2}$ Richard McCreery ${ }^{1,2}$ and Marek Malac ${ }^{1,3}$ \\ 1. Nanotechnology Research Center, Edmonton, Canada. \\ 2. Department of Chemistry, University of Alberta, Edmonton, Canada. \\ 3. Department of Physics, University of Alberta, Edmonton, Canada.
}

To visualize the internal components of electronic devices in three dimensions (3D), it is necessary to use an imaging method that provides high contrast difference between the materials the device is composed of. Often the contrast of bright field transmission electron microscope (BFTEM) or scanning transmission electron microscope (STEM) is too low resulting in high irradiation dose to obtain adequate signal to noise ratio (SNR) for reliable 3D reconstruction. The low contrast is of concern especially when the sample of interest is composed of light elements [1]. The high irradiation dose implies extensive radiation damage [2] and thus unreliable 3D volume reconstruction. High angle annular dark field (HAADF) STEM is not applicable as it relies on large difference of atomic number of the components. Core loss electron energy loss spectroscopy (EELS) typically requires high irradiation dose due to small core loss cross sections while low loss EELS signal delocalization is often of the same order as the lateral dimensions of the examined layers.

Hole-free phase plate (HFPP) provides contrast from (light element) samples with a small difference in atomic number and a small difference in mean inner potential $[3,4]$. The applicability of the HFPP with electron tomography has been demonstrated for biological specimens [5,6]. The contrast improvement provided by the phase plate was confirmed in projection images of thick $(400 \mathrm{~nm})$ biological samples. The applicability of HFPP to electron tomography of thick materials sciences samples is demonstrated here. The samples were prepared by a pluck-out in a focused ion beam (FIB) instrument. Rod shaped samples with 100 to $300 \mathrm{~nm}$ diameter were fabricated to allow collection of data sets without a missing wedge resulting in isotropic resolution in reconstructed $3 \mathrm{D}$ volume.

Figure $1 \mathrm{a}$ and $\mathrm{b}$ shows projected images extracted from a tilt series of a molecular electronics junction [6] without a) and with HFPP b). The interface between e-carbon and a bromophenyl oligomer can be clearly observed in Fig 1b) but cannot be detected at the same irradiation dose in a). We also measured the interface roughness using the reconstructed image $[7,8]$ shown in figure 1c. Figure 2 a shows a test sample of a multilayer sample composed of $\mathrm{Si} / \mathrm{C} / \mathrm{SiOx} / \mathrm{SiN}$ multilayer. The various layers can be clearly differentiated in the HFPP images. Figure $2 b$ shows the relation of the contrast for the various materials and sample rod diameter. The benefit in terms of contrast increase in HFPP imaging as compared to BFTEM increases as the sample rod diameter and atomic number decrease [9].

\section{References:}

[1] M. Hayashida et al, Micron 91 (2016), p. 49.

[2] R.F. Egerton et al, Micron 35 (2004), p. 399.

[3] M. Malac et al, Ultramicroscopy 118 (2012), p. 77.

[4] M. Malac et al, Micron 100 (2017), p. 10.

[5] R. Danev et al, PNAS 111 (2014), p. 15635.

[6] A. Morteza Najarian et al, ACS Nano 10 (2016), p. 8918. 
[7] Y. Fukuda et al, Journal of Structural Biology 190 (2015), p. 143.

[8] M. Hayashida et al, Proceedings of the 37the Annual NANO testing symposium (2016) p. 129.

[9] We acknowledge the help of Dr. Motoki, Dr. Konyuba, Dr. Ishikawa and Dr. Okura of JEOL Ltd.

(a)

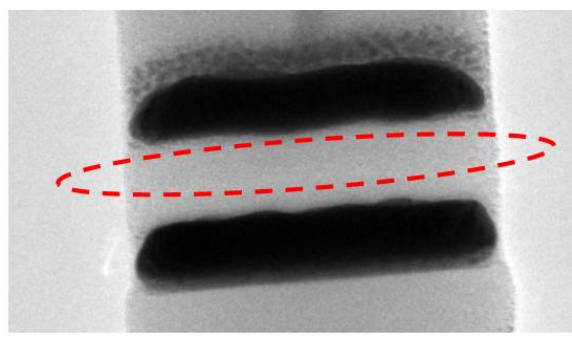

$50 \mathrm{~nm}$ (b)

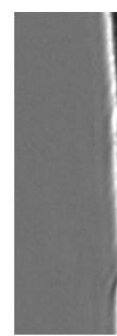

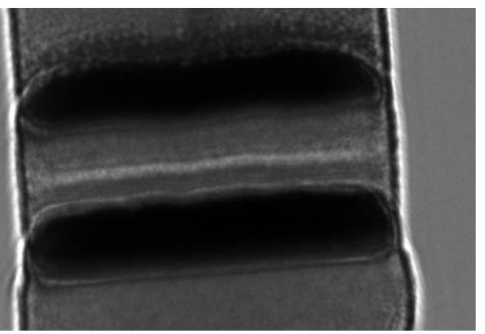

(c)

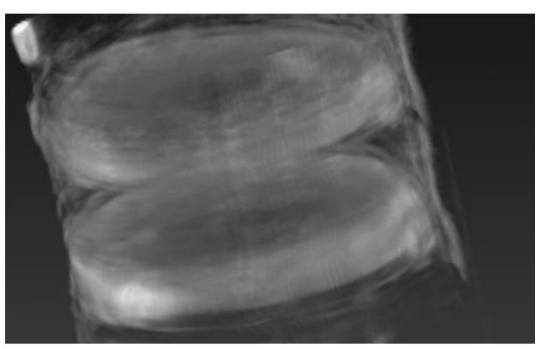

Figure 1. TEM images of molecular electronic junction with and without HFFP (a). While difference of contrast of eCarbon/Bromo-phenyl/ eCarbon layers in a circle is not visible at all without HFFP, the difference is clear with HFFP (b). Layer compositions from bottom to top are

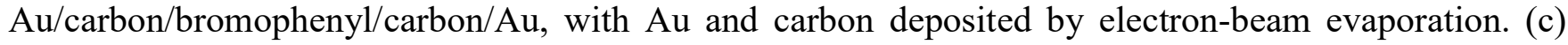
The reconstructed image.

(a)

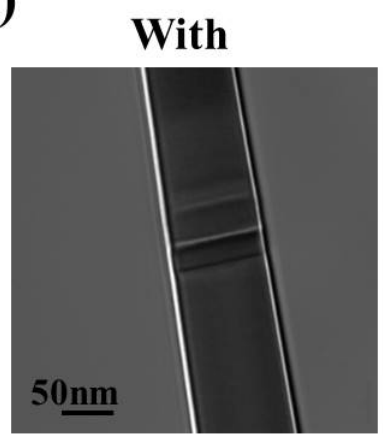

Without

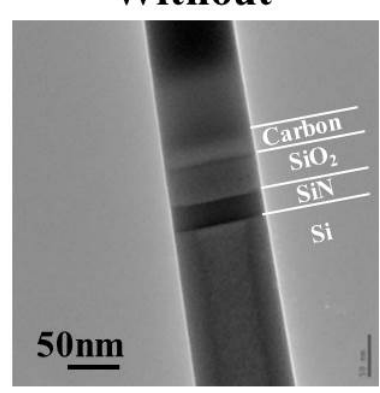

(b)

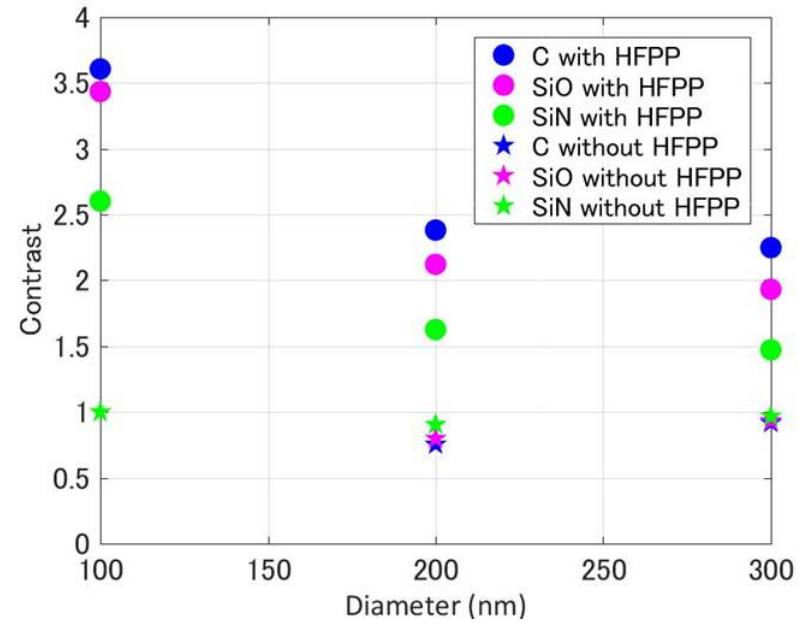

Figure 2. (a) TEM images of multilayer sample with $100 \mathrm{~nm}$ diameter with and without HFFP. (b) Relationship between contrast and thickness for each layer. (c) Reconstructed image. 